\title{
Research on virtual flux oriented synchronization algorithm for three-phase grid voltages
}

\author{
Lu Wang \\ Department of Mathematics and Information Engineering, Chongqing University of Education, Chongqing, \\ China \\ wanglu514@163.com
}

Keywords: three-phase grid voltages, synchronization algorithm, virtual flux orientation, filter, decoupling compensation.

\begin{abstract}
To deal with the synchronization of three-phase grid-connected converters, a synchronization algorithm based on virtual flux orientation is proposed in this paper. By introducing a low-pass filter and a high-pass filter, the orthogonal components of the virtual flux under the $\alpha \beta$ stationary coordinate frame that $\psi_{\alpha}$ and $\psi_{\beta}$ corresponding to the three-phase voltages can be calculated, and a decoupling compensation is adopted to eliminate the influence of these filters on the fundamental component of virtual flux. Then the phase angle of the three-phase grid voltages can be derived directly according to $\psi_{\alpha}$ and $\psi_{\beta}$, without any rotating coordinate transformation. The simulation results show that this algorithm can extract the phase angle of the fundamental component of grid voltage quickly and accurately under unideal power grid conditions, and it also has strong ability of frequency adaptation.
\end{abstract}

\section{Introduction}

When any kind of converter need to be connected to the power grid, the fundamental component of the grid voltage must be synchronously detected, which is used to control the grid-connected power converter and make it work in concert with the power grid. However, under unideal conditions, the grid voltage may be asymmetric, or under the influence of harmonics and changes in frequency and phase angle. All these unfavorable factors will affect the performance of the synchronization algorithm, and even cause overvoltage or overcurrent damage of grid connected converter [1]. Therefore, it is very important to find a synchronous algorithm for obtaining the fundamental components of the grid voltage quickly and accurately under various power grid conditions, which is of great significance for improving the steady and dynamic performance of grid-connected power converters and ensuring their safe and reliable operation.

The existing grid voltage synchronization algorithms can be generally divided into four kinds: zero crossing detection [2], discrete-fourier-transform-based [3], adaptive-filter-based [4] and phase-locked-loop (PLL). Among them, phase-locked-loop has high detection precision and fast dynamic response, it is the most widely used synchronization algorithm. The basic structure of a phase-locked loop is shown in Fig. 1, it consists of three fundamental blocks: the phase detector (PD), the loop filter (LF) and the voltage-controlled oscillator (VCO). PD generates an output signal proportional to the phase difference between the input signal $v$, and the signal generated by the internal oscillator of the PLL $v^{\prime}$, depending on the type of PD, high-frequency AC components appear together with the DC phase-angle difference signal. LF presents a low-pass filtering characteristic to attenuate the high-frequency AC components from the PD output, typically, this block is constituted by a first-order low-pass filter or a PI controller. VCO generates at its output an AC signal whose frequency is shifted with respect to a given central frequency $\omega_{c}$, as a function of the input voltage provided by the LF.

The LF block and the VCO block of all the PLL algorithms are almost the same respectively, and their differences usually lie in the PD block. From the PD, PLL can be divided into two categories: the first one is the PLL based on static reference coordinate frame, such as the PLL with 
the PD implemented by means of a simple multiplier [2,5], and an enhanced PLL(EPLL) using adaptive notch filter (ANF) proposed in [6], etc. the second one is the PLL based on synchronous reference frame (SRF-PLL). Fig. 2 shows a SRF-PLL based on the quadrature signal generator (QSG) [7], in which the PD block is constructed by QSG and Park transform, on the steady state, the rotation angle of the $d q$ coordinate frame, which is the output of the closed-loop, is equal to the phase angle of the input voltage $\theta$, and the d-axis component $v_{d}$ indicates the amplitude of the input voltage $v_{m}$. Because the outputs of the PD block are DC signals, the bandwidth of LPF can be set much bigger, and it has better dynamic performance compared with the PLL algorithm based on static reference frame. For an ideal three-phase system, the quadrature components under the $\alpha \beta$ stationary coordinate frame that $v_{\alpha}$ and $v_{\beta}$ can be easily obtained through the Clarke transform, and then fast and accurate detection of phase and amplitude of the grid voltage can be achieved. However, for the harmonic or three-phase unbalanced power grid, the detection result is not so good [5], to resolve these problems, a decoupled double synchronous reference frame PLL (DDSRF-PLL) and a PLL based on complex coefficient transfer function (CCF-Based PLL) are proposed in [8] and [9] respectively.

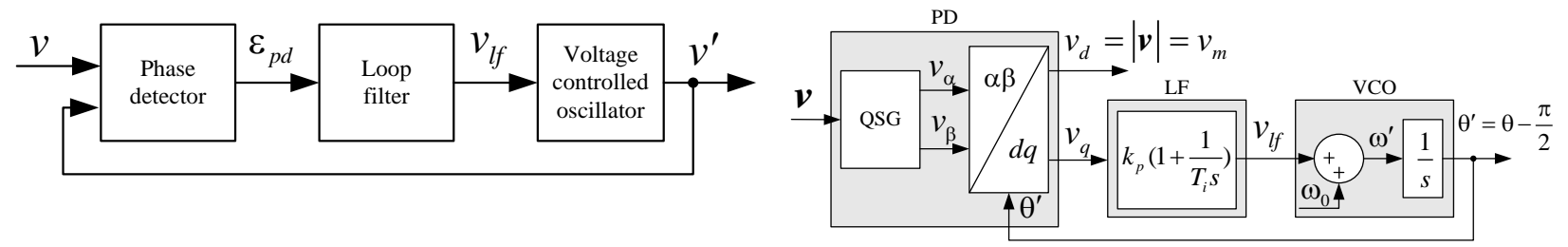

Figure 1 Basic structure of a PLL

Figure 2 PD based on QSG and Park transformation

In some cases, such as PWM rectifier, we don't need to know the specific phase value, as long as the sine and cosine values of phase angle should be used. Based on this point, this paper proposes a grid voltage synchronization algorithm without any rotating coordinate transformation.

\section{Principle of Virtual Flux Oriented Synchronization Algorithm}

Define the symmetrical three-phase grid voltages as

$$
\boldsymbol{U}_{a b c}=\left[\begin{array}{l}
u_{a} \\
u_{b} \\
u_{c}
\end{array}\right]=\left[\begin{array}{l}
U_{m} \cos \left(\omega_{0} t+\varphi\right) \\
U_{m} \cos \left(\omega_{0} t-2 \pi / 3+\varphi\right) \\
U_{m} \cos \left(\omega_{0} t+2 \pi / 3+\varphi\right)
\end{array}\right]
$$

Where $U_{m}, \omega_{0}, \varphi$ represent the amplitude, the angular velocity and the initial phase-angle respectively.

(1) can be expressed in the stationary reference frame as

$$
\boldsymbol{U}_{a \beta}=T_{a b c / \alpha \beta} \boldsymbol{U}_{a b c}==\left[\begin{array}{l}
u_{\alpha} \\
u_{\beta}
\end{array}\right]=\left[\begin{array}{c}
U_{m} \cos \left(\omega_{0} t+\varphi\right) \\
U_{m} \sin \left(\omega_{0} t+\varphi\right)
\end{array}\right]
$$

Where $\boldsymbol{T}_{a b c / a \beta}$ denotes the Clarke transform matrix given by

$$
T_{a b c / a \beta}=\frac{2}{3}\left[\begin{array}{ccc}
1 & -\frac{1}{2} & -\frac{1}{2} \\
1 & \frac{\sqrt{3}}{2} & -\frac{\sqrt{3}}{2}
\end{array}\right]
$$

Then from (2), the phase angle of the grid voltage can be obtained as 


$$
\left\{\begin{array}{l}
\cos \theta=\cos \left(\omega_{0} t+\varphi\right)=\frac{u_{\alpha}}{\sqrt{u_{\alpha}{ }^{2}+u_{\beta}{ }^{2}}} \\
\sin \theta=\sin \left(\omega_{0} t+\varphi\right)=\frac{u_{\beta}}{\sqrt{u_{\alpha}{ }^{2}+u_{\beta}^{2}}}
\end{array}\right.
$$

Because in the above method, the phase angle of the grid voltage is directly calculated according to the instantaneous voltage, However, the grid voltage may contain harmonics and other disturbances, in this case, the detection accuracy for the phase of the fundamental voltage vector will be significantly reduced, furthermore, the control performance of the whole system will become worse.

Based on the related theory of motor control, a virtual flux vector can be constructed as follow

$$
\boldsymbol{U}_{a b c}=d \boldsymbol{\psi}_{a b c} / d t
$$

According to (1) and (2), the phase angle of three-phase grid voltage $\theta$ can be derived as

$$
\left\{\begin{array}{l}
\cos \gamma=\frac{\psi_{\alpha}}{\sqrt{\psi_{\alpha}^{2}+\psi_{\beta}^{2}}} \\
\sin \gamma=\frac{\psi_{\beta}}{\sqrt{\psi_{\alpha}^{2}+\psi_{\beta}^{2}}} \\
\gamma=\theta-\frac{\pi}{2}
\end{array}\right.
$$

Where $\gamma$ represents the phase angle of the virtual flux vector $\psi_{a b c}$.

Due to the use of integral operation, which has the characteristic of low-pass filtering, in the process of solving the virtual flux, the influence of voltage harmonics on the flux can be effectively overcome, and the accuracy of the synchronization algorithm can be ensured. However, there is usually zero drift in the detection circuit and the initial value of the measured signal is not zero, so these DC components will cause the problem of integral saturation if only a pure integrator is used. For this reason, the first-order low-pass filter (LPF) as shown in (6) can be used to replace the pure integrator, which can completely suppress the DC component problem caused by the initial value.

$$
G_{L P F}(s)=\frac{1}{s+\omega_{c}}
$$

Where $\omega_{c}$ is the cut-off frequency of LPF.

In order to meet the requirement of response speed, the cut-off frequency of the low-pass filter can't be set too low, therefore, a simple first-order low-pass filter can't completely suppress the DC component, so a high-pass filter (HPF) should be added, and the transfer function from the voltage vector to the virtual flux vector can be derived as

$$
\boldsymbol{G}(s)=\frac{\boldsymbol{\psi}_{a b c}^{\prime}(s)}{\boldsymbol{U}_{a b c}(s)}=\frac{1}{s+k_{1} \omega_{0}} \frac{s}{s+k_{2} \omega_{0}}
$$

Where $k_{1}$ and $k_{2}$ are the frequency coefficients.

It is obvious that the DC gain of $\boldsymbol{G}(s)$ is zero, but the amplitude of the fundamental voltage is weakened and the phase angle is offset through these filters. Therefore, it is necessary to make further compensation.

The steady-state phasors corresponding to the fundamental components can be expressed as

$$
\dot{\psi}_{a b c}^{\prime}=\frac{1}{j \omega_{0}+k_{1} \omega_{0}} \frac{j \omega_{0}}{j \omega_{0}+k_{2} \omega_{0}} \dot{\boldsymbol{U}}_{a b c}=\frac{-1}{\left(j+k_{1}\right)\left(j+k_{2}\right)} \frac{\dot{\boldsymbol{U}}_{a b c}}{j \omega_{0}}=\frac{-1}{\left(j+k_{1}\right)\left(j+k_{2}\right)} \dot{\boldsymbol{\psi}}_{a b c}
$$


And

$$
\left\{\begin{array}{l}
\dot{\psi}_{a b c}^{\prime}=\psi_{\alpha}^{\prime}+j \psi_{\beta}^{\prime} \\
\dot{\psi}_{a b c}=\psi_{\alpha}+j \psi_{\beta}
\end{array}\right.
$$

Substituting (9) into (8), the components of virtual flux on the stationary reference frame can be obtained as

$$
\left\{\begin{array}{l}
\psi_{\alpha}=\left(1-k_{1} k_{2}\right) \psi_{\alpha}^{\prime}+\left(k_{1}+k_{2}\right) \psi_{\beta}^{\prime} \\
\psi_{\beta}=\left(1-k_{1} k_{2}\right) \psi_{\beta}^{\prime}-\left(k_{1}+k_{2}\right) \psi_{\alpha}^{\prime}
\end{array}\right.
$$

From all above, the virtual flux oriented synchronization algorithm for three-phase grid voltage is shown in Fig. 3.

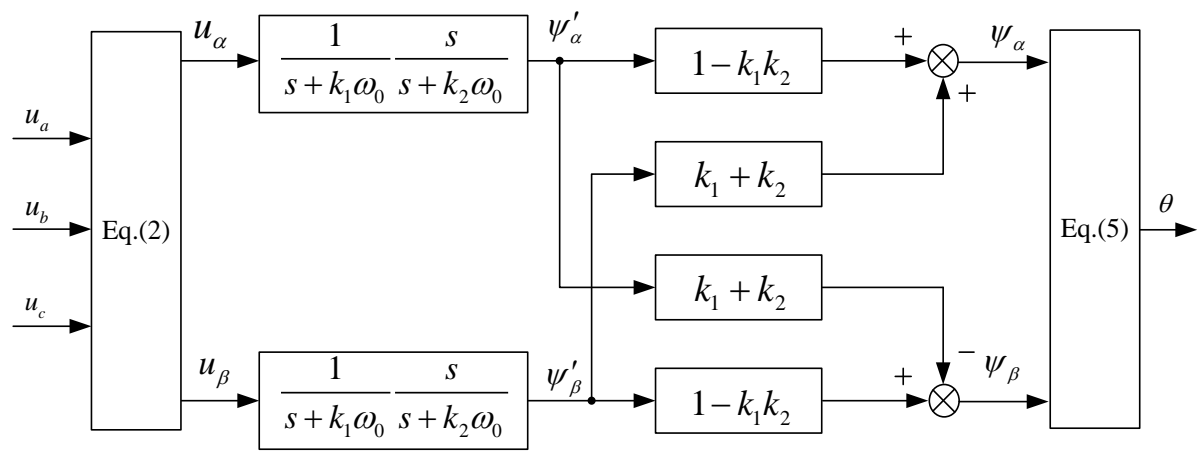

Figure 3 Virtual flux oriented synchronization algorithm for three-phase grid voltages

\section{Tuning of the Control Parameters}

The cut-off frequency $\omega_{c}$ in (6) directly affects the dynamic response performance of the system. If $\omega_{c} / \omega_{0}>0.707$, the greater the ratio $\omega_{c} / \omega_{0}$, the faster the system responses, but the larger the overshoot and the longer the dynamic oscillation time. If $\omega_{c} / \omega_{0}<0.707$, the system overshoot will be smaller, however, the smaller the ratio $\omega_{c} / \omega_{0}$, the slower the system responses. In this paper, the dynamic response speed and the stability of the system are both taken into consideration, the frequency coefficient $k_{1}$ is set to 0.707 .

From the transfer function shown in (7), it is obvious that the value of $k_{2}$ does not affect the effect on the suppression of DC component, but only plays a decisive role on the response speed and the attenuation effect on the AC components. The greater $k_{2}$, the faster the system responses, but the larger the overshoot and the greater the attenuation of the fundamental component. Fortunately, by dividing the attenuation ratio, this attenuation can be compensated at the expense of amplifying the high-order harmonic components. Therefore, it is necessary to compromise these two factors for the selection of the frequency coefficient, and $k_{2}$ is set to 0.67 in this paper.

Under the conditions of $k_{1}=0.707$ and $k_{2}=0.67$, the step response of the transfer function expressed in (7) is shown in Fig. 4, and the response time is about 0.025 seconds.

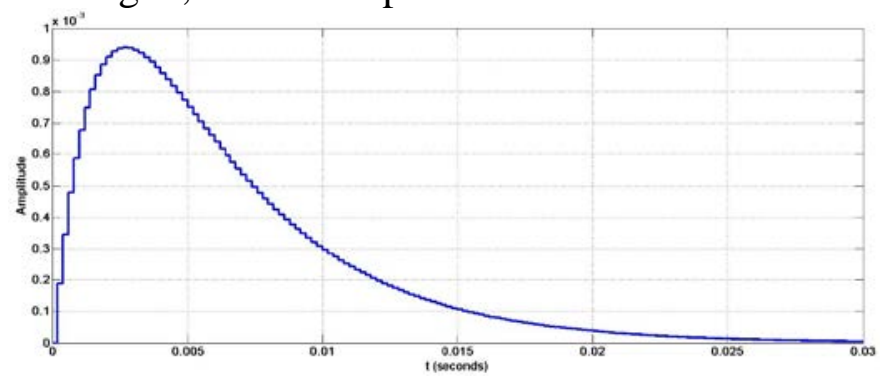

Figure 4 Step response of the transfer function expressed in (7) 


\section{Simulation and Analysis}

When the amplitude of the three-phase reference voltage is $311 \mathrm{~V}$ and the frequency is $50 \mathrm{~Hz}$, $52 \mathrm{~Hz}$, or $48 \mathrm{~Hz}$, the outputs of PLL are shown in Fig. 5, Fig. 6, and Fig. 7 respectively. Therein, $U_{a-b a s e}, U_{b-b a s e}$ and $U_{c-b a s e}$ are the fundamental components of the three-phase reference voltages, $U_{a o}$, $U_{b o}$ and $U_{c o}$ are the output voltages of the PLL accordingly, $\theta_{b a s e}$ is the phase angle of the reference voltage of phase $A$, and $\theta$ is the output of the PLL corresponding to $\theta_{\text {base }}$. It can be seen that the algorithm described in this paper can adapt to frequency change, in these three cases, the synchronous tracking time is less than 0.02 seconds.

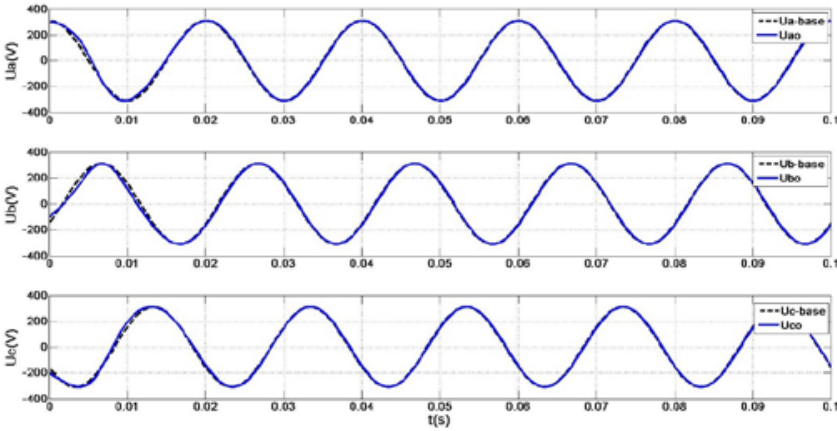

(a) Three-phase voltages

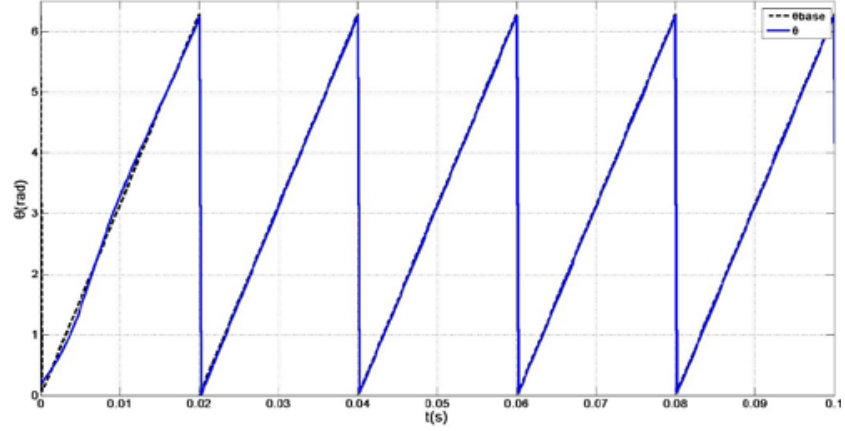

(b) Phase angle of the voltage of phase $\mathrm{A}$

Figure 5 Synchronous tracking result of PLL when $f_{0}=50 \mathrm{~Hz}$

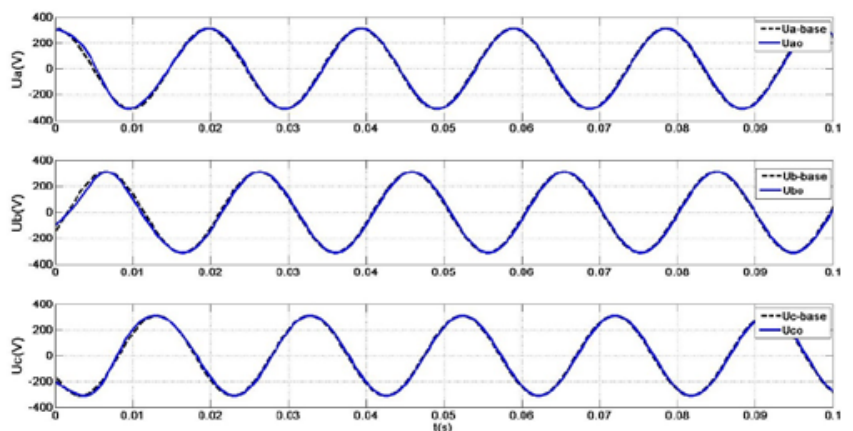

(a) Three-phase voltages

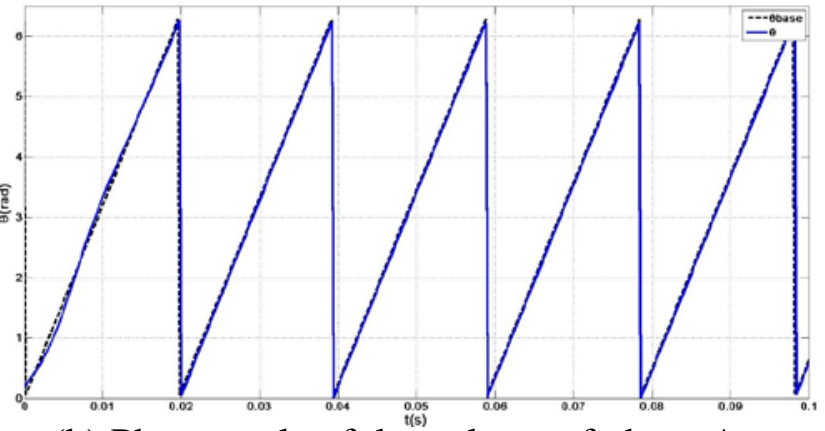

(b) Phase angle of the voltage of phase $\mathrm{A}$

Figure 6 Synchronous tracking result of PLL when $f_{0}=52 \mathrm{~Hz}$

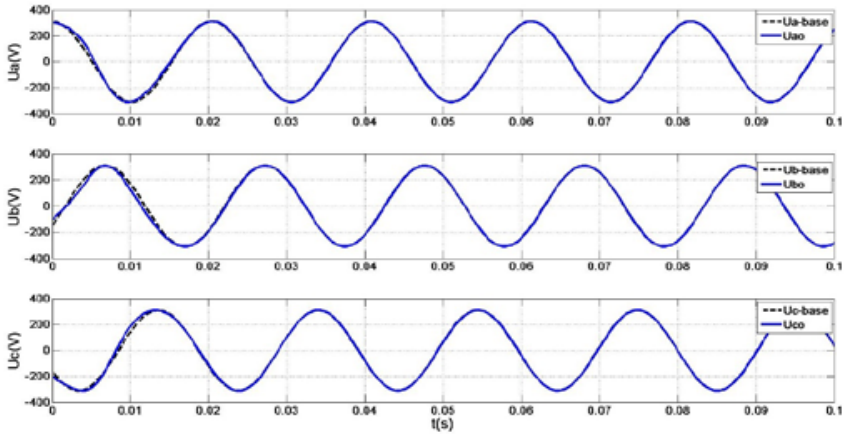

(a) Three-phase voltages

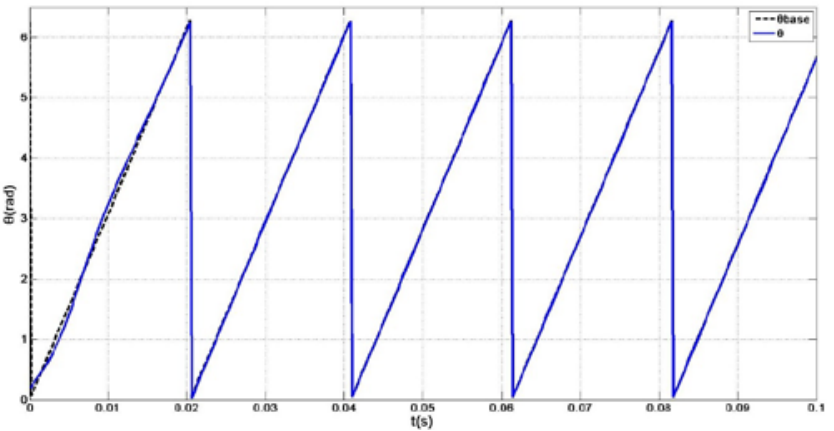

(b) Phase angle of the voltage of phase A

Figure 7 Synchronous tracking result of PLL when $f_{0}=48 \mathrm{~Hz}$

In order to test the harmonic suppression ability of the algorithm, supposing the fundamental component of the reference voltage as a sinusoidal waveform with the amplitude of $311 \mathrm{~V}$ and the frequency of $50 \mathrm{~Hz}$, and superimposing a five-order positive sequence component and a seven-order negative sequence component on it, the harmonic content is $4 \%$ and $2 \%$ respectively. The simulation result is shown in Fig. 8, it can be seen that the algorithm described in this paper has a strong ability to suppress harmonics. 


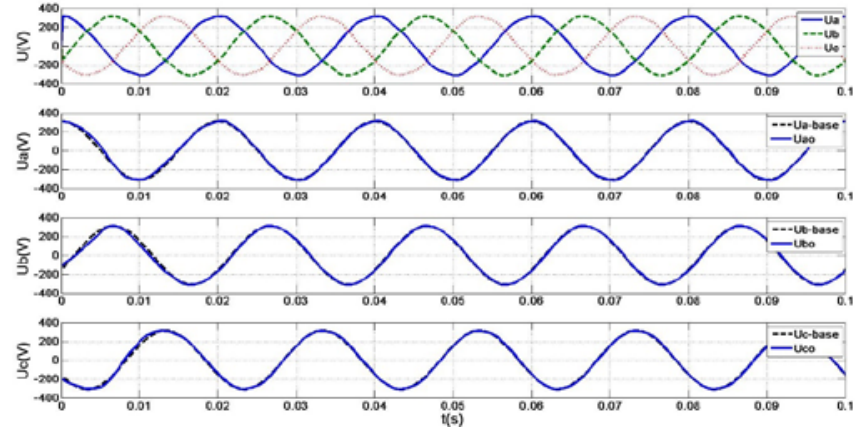

(a) Three-phase voltages

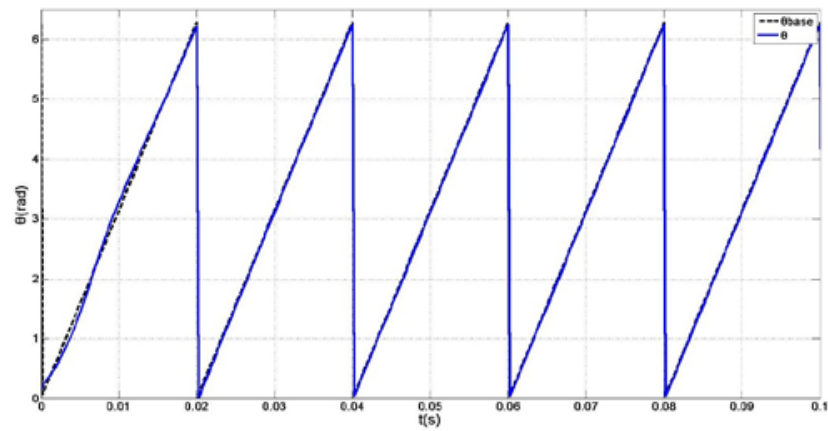

(b) Phase angle of the voltage of phase A

Figure 8 Synchronous tracking result on harmonic suppression

\section{Conclusion}

This paper constructs a three-phase voltage synchronization system based on virtual flux orientation. The principle and parameter design method of synchronization algorithm are introduced. The simulation results show that this algorithm can better adapt to the change of the frequency, and can restrain harmonics, synchronization tracking time is less than 0.02 seconds. It can be widely used in the new energy grid-connected system, modular parallel uninterruptible power supply, and other occasions with high demands for tracking grid voltage.

\section{Acknowledgements}

This work was supported by the Natural Science Foundation of Chongqing of China under Grant (No. KJ1501406).

\section{References}

[1] Golestan S, Monfared M, Freijedo F. Design-oriented study of advanced synchronous reference frame phase-locked loops[J]. IEEE Transactions on Power Electronics, 2013, 28(2): 765-778.

[2] Geng H, Xu D, Wu B. A novel hardware-based all-digital phase-locked loop applied to gridconnected power converters[J]. IEEE Transactions on Industrial Electronics, 2011, 58(5):1737-1745.

[3] Kong X J, Luo F, Peng L, et al. Implement and parameter design of DPLL for invertors based on cycle control[J]. Proceedings of the CSEE, 2007, 27(1):60-64.

[4] Rodriguez P, Luna A, Candela I, et al. Multiresonant frequency-locked loop for grid synchronization of power converters under distorted grid conditions[J]. IEEE Transactions on Industrial Electronics, 2011,58(1):127-138.

[5] Chung S. A phase tracking system for three phase utility interface inverters[J]. IEEE Transactions on Power Electronics, 2000, 15(3):431-438.

[6] Karimi-Ghartemani M, Iravani M R. A nonlinear adaptive filter for online signal analysis in power systems: Applications[J]. IEEE Transactions on Power Delivery, 2002, 17(2):617-622.

[7] Xiao F R, Dong L, Li L, et al. A frequency-fixed SOGI-based PLL for single-phase gridconnected converters[J]. IEEE Transactions on Power Electronics, 2017, 32(3):1713-1819.

[8] Rodriguez P, Pou J, Bergas J, et al. Decoupled double synchronous reference frame PLL for power converters control[J]. IEEE Transactions on Power Electronics, 2007, 22(2):584-592.

[9] Guo X, Wu W, Chen Z. Multiple-complex coefficient-filter-based phase-locked loop and synchronization technique for three phase grid-interfaced converters in distributed utility networks[J]. IEEE Transactions on Industrial Electronics, 2011, 58(4):1194-1204. 